\title{
Con respecto al diagnóstico de pancreatitis crónica
}

\section{With regard to the diagnosis of chronic pancreatitis}

Gilber Jesús Quispe-Cobián, ${ }^{1}$ Diana Karen Quispe-Larrota. ${ }^{*}$

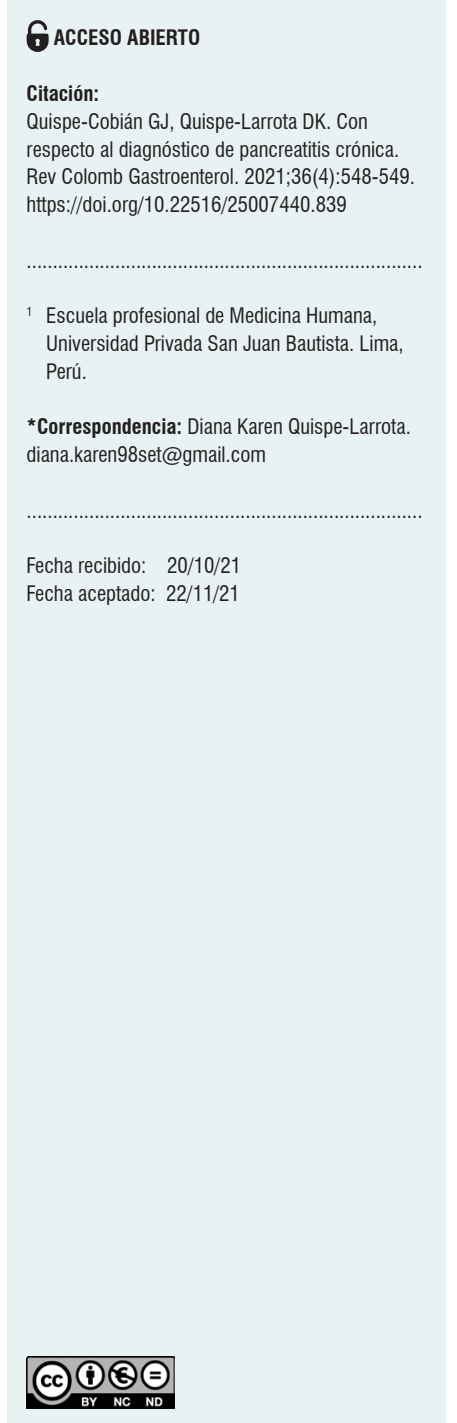

Señor editor:

Hemos leído detenidamente el artículo titulado "Descripción clínica y epidemiológica de pacientes con pancreatitis crónica en un hospital de alta complejidad en Cali" que describe los criterios clínicos para el diagnóstico de pancreatitis crónica.

La presente tiene como objetivo sustentar en los siguientes puntos porque consideramos que dicho artículo no ha tenido el planteamiento más adecuado.

En primer lugar, consideramos que falta describir criterios más específicos, que representan un valor de suma importancia para el diagnóstico de pancreatitis crónica según la clasificación japonesa, como se describe claramente en el artículo de Sheel y colaboradores:

- Criterios definitivos:

- Hallazgos de imagen: calcificaciones, cálculos, cambios en la morfología ductal.

- Histológicos: pérdida del parénquima exocrino con fibrosis a predominio interlobulillar.

- Criterios sugestivos de pancreatitis temprana: dolor abdominal superior repetido, enzimas pancreáticas elevadas, insuficiencia exocrina, consumo excesivo de alcohol continuo $>80 \mathrm{~g} / \mathrm{día}^{(1)}$.

Asimismo, creemos que describir la incidencia de pancreatitis crónica hasta la fecha en Cali, Colombia, según el grupo etario, ayudaría a tener una perspectiva mas amplia de las características de la enfermedad.

En segundo lugar, se describen siglas resumidas correspondientes con los criterios de la Clasificación internacional de enfermedades, 10. a edición (CIE-10). Especificar el nombre del diagnóstico aumentaría la practicidad de la lectura y la rapidez de interiorización de la información para aquellos lectores que no dominan totalmente la CIE-10.

En tercer lugar, se indica la importancia de valorar las condiciones asociadas y factores de riesgo para el diagnóstico temprano de pancreatitis crónica, en concordancia con lo expuesto por Arango M y colaboradores; en algunos pacientes en los que se pueden observar cambios discretos en su analítica, pero cuyos síntomas no se explican con los criterios típicos es importante valorar la historia clínica y los diversos factores $\operatorname{asociados}^{(2)}$. Creemos que es una indicación incompleta ya que, ante la asociación de dichos factores y datos sugestivos, pero no concluyentes luego de una tomografía axial computarizada (TAC), la actuación correcta seria indicar posteriormente 
una colangiopancreatografía por resonancia magnética (CPRM), puesto que su mayor sensibilidad ayudaría a detectar cambios más específicos, tanto ductales como parenquimatosos, como lo indican Frøkjær y colaboradores $^{(3)}$, y de este modo lograr un diagnóstico temprano de pancreatitis crónica más eficaz.

En cuarto lugar, inicialmente se identificaron 97 pacientes, de los cuales solo 36 fueron aceptados con los criterios de inclusión. Esta cifra reducida disminuye la confiablidad de los resultados. Consideramos que sería oportuno indicar claramente esto en su manuscrito para no crear sesgos en la divulgación de la información.
Finalmente, se compararon las características que se tuvieron en cuenta en el presente artículo con otros estudios internacionales; sin embargo, no se describen cuáles fueron las características a nivel internacional, sexo predominante, edad o raza. Por tanto, se observa como un error comparar este estudio frente a estudios internacionales, porque no existen citas que lo sustenten en la introducción o en general. Además, los resultados no pueden crear escalas de estadios tempranos, ya que existen diversas clasificaciones, como lo son Lees-Wiersema, clasificación japonesa, clasificación de Rosemont y clasificación de Milwaukee, que ya se encuentran definidas ${ }^{(2)}$.

\section{REFERENCIAS}

1. Sheel ARG, Baron RD, Sarantitis I, Ramesh J, Ghaneh P, Raraty MGT, Yip V, Sutton R, Goulden MR, Campbell F, Farooq A, Healey P, Jackson R, Halloran CM, Neoptolemos JP. The diagnostic value of Rosemont and Japanese diagnostic criteria for 'indeterminate', 'suggestive', 'possible' and 'early' chronic pancreatitis. Pancreatology. 2018;18(7):774-784. https://doi.org/10.1016/j.pan.2018.08.002

2. Arango Molano LA, Díaz Tobar CP, Caicedo Q. CA, Angel Rodríguez C. Estado actual del diagnóstico y manejo de la pancreatitis crónica. Rev Colomb Gastroenterol. 2019;34(4):376-84.
3. Frøkjær JB, Akisik F, Farooq A, Akpinar B, Dasyam A, Drewes AM, Haldorsen IS, Morana G, Neoptolemos JP, Olesen SS, Petrone MC, Sheel A, Shimosoegawa T, Whitcomb DC; Working group for the International (IAP - APA - JPS - EPC) Consensus Guidelines for Chronic Pancreatitis. Guidelines for the Diagnostic Cross Sectional Imaging and Severity Scoring of Chronic Pancreatitis. Pancreatology. 2018;18(7):764-773.

https://doi.org/10.1016/j.pan.2018.08.012 